\title{
Two cases of fungal cyst infection in ADPKD: is this really a rare complication?
}

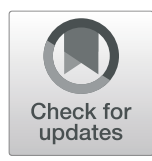

\author{
Laura Onuchic ${ }^{1}$ (D), Victor Augusto Hamamoto Sato ${ }^{2}$, Precil Diego Miranda de Menezes Neves ${ }^{1}$, \\ Bruno Eduardo Pedroso Balbo ${ }^{1}$, Antônio Abel Portela-Neto ${ }^{1}$, Fernanda Trani Ferreira', Elieser Hitoshi Watanabe ${ }^{1}$, \\ Andreia Watanabe ${ }^{1}$, Maria Cláudia Stockler de Almeida ${ }^{3}$, Leonardo de Abreu Testagrossa ${ }^{4}$, \\ Pedro Renato Chocair ${ }^{2}$ and Luiz Fernando Onuchic ${ }^{1 *}$
}

\begin{abstract}
Background: Cyst infection is a prevalent complication in autosomal dominant polycystic kidney disease (ADPKD) patients, however therapeutic and diagnostic approaches towards this condition remain unclear. The confirmation of a likely episode of cyst infection by isolating the pathogenic microorganism in a clinical scenario is possible only in the minority of cases. The available antimicrobial treatment guidelines, therefore, might not be appropriate to some patients.

Case presentation: We describe two unique cases of kidney cyst infection by Candida albicans, a condition that has not been previously described in literature. Both cases presented clear risk factors for Candida spp. infection. However, since there was no initial indication of cyst aspiration and culture, antifungal therapy was not immediately started and empirical treatment was initiated as recommended by the current guidelines. Antifungal treatment was instituted in both cases along the clinical course, according to their specificities.

Conclusion: Our report highlights the possibility of Candida spp. cyst infection. Failure of clinical improvement with antibiotics should raise the suspicion of a fungal infection. Identification of infected cysts should be pursued in such cases, particularly with PET-CT, and when technically possible followed by cyst aspiration and culture to guide treatment. Risk factors for this condition, such as Candida spp. colonization, previous antimicrobial therapy, hemodialysis, necrotizing pancreatitis, gastrointestinal/hepatobiliary surgical procedure, central venous catheter, total parenteral nutrition, diabetes mellitus and immunodeficiency (neutropenia $<500$ neutrophils $/ \mathrm{mL}$, hematologic malignancy, chemotherapy, immunosuppressant drugs), should be also considered accepted criteria for empirical antifungal therapy.
\end{abstract}

Keywords: Autosomal dominant polycystic kidney disease, Cyst infection, Fungal infection, Candida albicans, Antifungal treatment

\section{Background}

Autosomal dominant polycystic kidney disease (ADPKD) is the most common monogenic kidney disorder, affecting approximately 1 in 1000 individuals [1-3]. Urinary tract infections (UTI) are among the most prevalent complications in this disease, occurring in $30-50 \%$ of the patients throughout their lifetime $[4,5]$. Renal cyst infection $(\mathrm{CI})$ is of particular interest in this scenario, since it is associated

\footnotetext{
* Correspondence: lonuchic@usp.br

${ }^{1}$ Department of Medicine, Division of Nephrology, University of São Paulo School of Medicine, Avenida Doutor Arnaldo, 455 - Sala 4304, São Paulo, SP 01246-903, Brazil

Full list of author information is available at the end of the article
}

with significant morbidity and mortality [6, 7]. Gramnegative bacteria are the typical causing agents in these episodes, most often related to the ascending urinary tract route $[8,9]$. Interestingly, the urine sediment may be bland and urine culture negative in $\mathrm{CI}$, because some cysts are not contiguous to the collecting system $[1,10]$. While the diagnosis of this condition may be difficult, in recent years positron emission tomography/computed tomography (PET-CT) has become the most sensitive imaging technique to establish it $[1,3,8,11]$.

Although less usually, alternative etiologic agents and infectious routes have been also associated with renal CI

(c) The Author(s). 2019 Open Access This article is distributed under the terms of the Creative Commons Attribution 4.0 International License (http://creativecommons.org/licenses/by/4.0/), which permits unrestricted use, distribution, and reproduction in any medium, provided you give appropriate credit to the original author(s) and the source, provide a link to the Creative Commons license, and indicate if changes were made. The Creative Commons Public Domain Dedication waiver (http://creativecommons.org/publicdomain/zero/1.0/) applies to the data made available in this article, unless otherwise stated. 
in several reports $[10,12]$. Fungal $\mathrm{CI}$, however, is recognized as a rare event in ADPKD, with very limited prior description [13, 14]. Notably, in this report we present two cases of fungal $\mathrm{CI}$ with distinct outcomes, bringing attention to Candida albicans as a potential causative agent in $\mathrm{CI}$ and to the possibility that this complication be more common than currently assumed.

\section{Case presentation}

\section{Case 1}

A 34-year-old female with ADPKD was referred for persistent fever and malaise for the past 33 days. She reported symptoms and signs consistent with vaginal candidiasis 2 months prior to admission followed by right kidney obstruction secondary to ureteral calculus, which led to double-J stent placement. Two days after the procedure she developed fever of $101^{\circ} \mathrm{F}$ and diffuse right abdominal pain, still sustained at admission. No urinary or gastrointestinal symptoms were reported. Urine and blood cultures were negative; white blood cell count and C-reactive protein (CRP), however, were elevated $\left(14,650 / \mathrm{mm}^{3}\right.$ and $389 \mathrm{mg} / \mathrm{L}$, respectively). Initial computed tomography (CT) was inconclusive for CI. The patient was placed on broad-spectrum antibiotic regimens for the following 2 months, including intravenous (IV) ciprofloxacin $500 \mathrm{mg}$ bid for 5 days, IV cephepime $1 \mathrm{~g}$ bid for 6 days, IV imipenem $500 \mathrm{mg}$ bid plus metronidazole $500 \mathrm{mg}$ tid for 20 days, and IV meropenem $1 \mathrm{~g}$ tid plus vancomycin $1 \mathrm{~g}$ bid plus fluconazole $200 \mathrm{mg}$ sid for 4 days. Despite this treatment, she displayed no clinical improvement and developed acute kidney injury likely secondary to unresolved infection and potentially to vancomycin nephrotoxicity, with a rise in serum creatinine from 0.38 to $2.3 \mathrm{mg} / \mathrm{dL}$. The laboratory tests are summarized in Table 1.

Once transferred to our center, the patient was submitted to a positron emission tomography-computed tomography (PET-CT) scan (Fig. 1a) which revealed high F-18 Fluor-deoxi-glucose (FDG) uptake in multiple right kidney cysts (Fig. 1b). Ultrasound-guided percutaneous drainage of the dominant suspected cyst followed by direct Grocott's methenamine silver staining of the cyst content led to the diagnosis of fungal elements consistent with Candida spp., followed by isolation of Candida albicans from culture of the collected material. She was then immediately restarted on IV fluconazole 200 mg sid. During a 10-day period of this therapy, however, the patient maintained fever and malaise, associated with weight loss, progressive decline in renal function and very high levels of serum C-reactive protein (153-310 $\mathrm{mg} / \mathrm{L}$, for a normal reference range below $5 \mathrm{mg} / \mathrm{L}$ ), with no tendency towards improvement. Given the refractoriness to this treatment, she was submitted to right nephrectomy (Fig. 1c, an estimated 1380-mL kidney), which led to renal failure and initiation of hemodialysis. Histopathology analysis confirmed this finding, showing cystic and pericystic hypha and pseudohypha invasion (Fig. 1d-f). At that moment she presented an unexpected tonic-clonic seizure not related to a significant electrolytic or hemodynamic disbalance but instead to a non-aneurysmatic subarachnoid hemorrhage documented by $\mathrm{CT}$, which prompted her transfer to the intensive care unit.

Prolonged hospitalization led to urinary infection by carbapenemase-resistant Klebsiella pneumoniae followed by septic shock. An over two-week therapy with broadspectrum antibiotics (IV tigecycline $50 \mathrm{mg}$ bid plus ertapenem $1 \mathrm{~g}$ sid plus meropenem $1 \mathrm{~g}$ bid plus PO phosphomycin $3 \mathrm{~g}$ sid each 3 days) led to no clinical improvement and a new CT revealed multiple left kidney cysts with enhanced wall thickening. The patient eventually required high doses of vasopressors. In this context, left nephrectomy was performed (1093-mL kidney), resulting in clinical improvement. When recovering from surgery, however, she presented left-lower-limb deep venous thrombosis, requiring systemic anticoagulation. Critical falls in hemoglobin levels during several attempts of this intervention, in turn, made necessary the placement of a cava vein filter. After a debilitating eight-month period, she was discharged to her local medical facility for chronic hemodialysis still presenting significant frailty. Two months later, however, she died following a diagnosis of acute pancreatitis.

Table 1 Summary of the main patients' laboratory tests

\begin{tabular}{lll}
\hline Laboratory Tests & Case 1 & Case 2 \\
\hline Serum Creatinine at admission & $2.3 \mathrm{mg} / \mathrm{dL}$ & $2.3 \mathrm{mg} / \mathrm{dL}$ \\
White Blood Cell Count at admission & $14,650 / \mathrm{mm}^{3}$ & $9040 / \mathrm{mm}^{3}$ \\
Reactive C-Protein at admission & $389 \mathrm{mg} / \mathrm{L}$ & $102.9 \mathrm{mg} / \mathrm{L}$ \\
Urine Leucocytes at admission & $>100 /$ field & $49,000 / \mathrm{mL}$ \\
Urine Red Blood Cells at admission & $>100 /$ field & $5000 / \mathrm{mL}$ \\
Blood Culture & Negative & Negative \\
Urine Culture & Candida albicans & Negative \\
Cyst Aspiration Culture & Candida albicans & Candida albicans \\
\hline
\end{tabular}




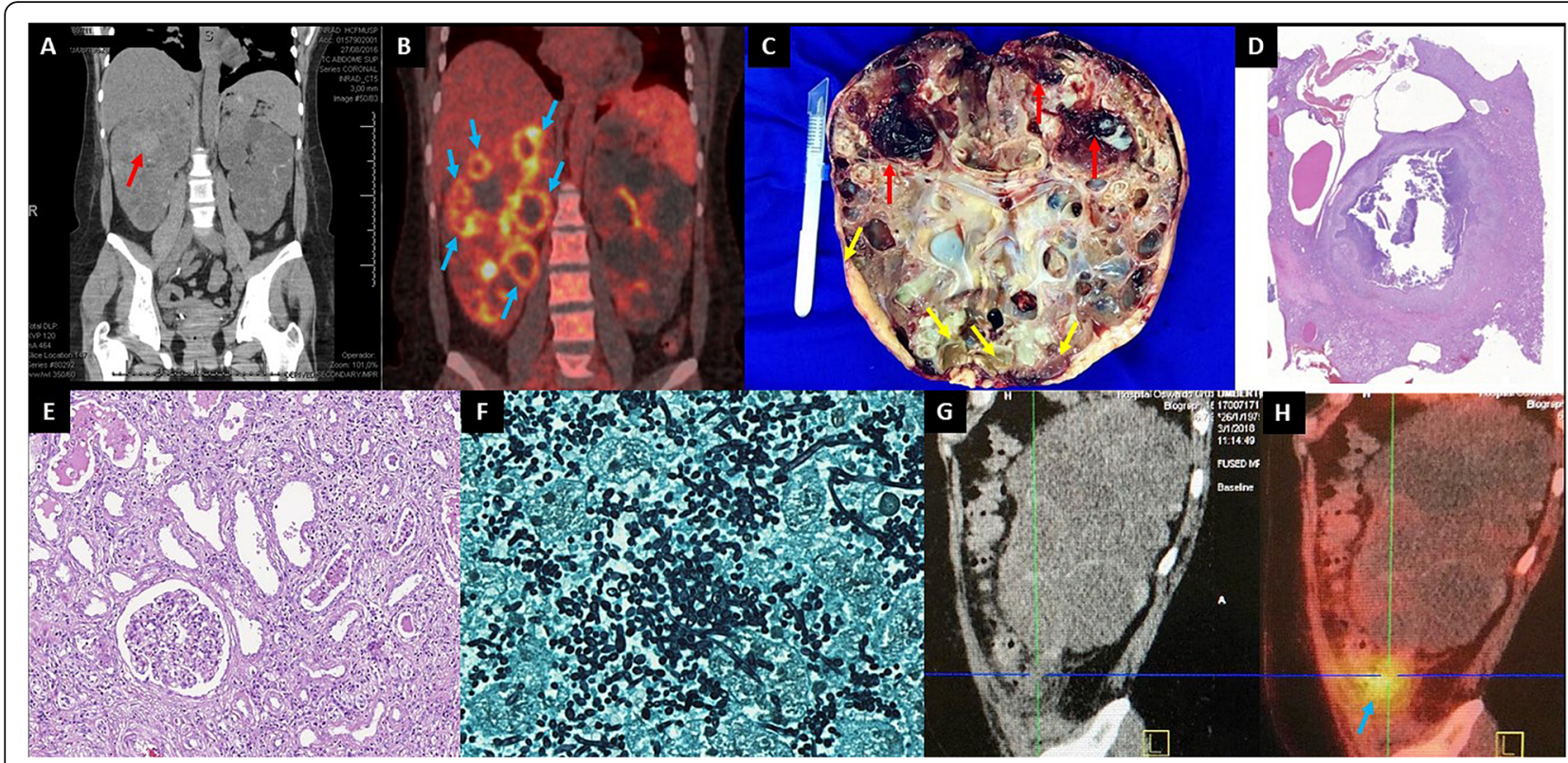

Fig. 1 A prior study [11] revealed that ultrasound, CT and magnetic resonance imaging (MRI) failed to detect the infected cysts in 94, 82 and 60\% of the cases, respectively. PET-CT, on the other hand, showed sensitivity of 100\% on the basis of FDG uptake by inflammatory cells. Such data are in agreement with a previous report of ours [10]. Both of our cases support such a diagnostic capacity of PET-CT. a Non-contrasted computed tomography (CT) scan on coronal view shows no evidence of cyst infection. Some cysts present higher density, a finding suggestive of recent hemorrhage (red arrow); b Positron emission tomography-computed tomography imaging: coronal section analysis reveals increased cyst-lining 18-FDG uptake activity (blue arrows), a very suggestive finding of cyst infection; c Gross appearance of the right kidney after nephrectomy. This enlarged kidney presented multiple medium and large-sized cysts filled with pus (yellow arrows) or blood (red arrows); Histologic analysis of kidney section evidenced $\mathbf{d}$ an area of inflammatory cavitation, centered on urinary tract and adjacent to some cysts on the left superior field (hematoxylin-eosin, $\times 4$ obj.); e Kidney parenchyma with neutrophilic interstitial infiltration, acute tubular damage and tubular neutrophilic casts (hematoxylin-eosin, $\times 20$ obj.); f Numerous septate hyphae and yeast microorganisms with morphological features of Candida sp., positively stained by Grocott's methenamine silver ( $\times 100$ obj.); $\mathbf{g}$ Sagital view of enlarged kidneys with multiple cysts containing homogeneous liquid or heterogeneous dense hyperproteic material; $\mathbf{h}$ Imaging assessment reveals perirenal fascia thickening and high FDG uptake in an exophytic cyst (blue arrow), yielding the diagnosis of renal cyst infection

\section{Case 2}

A 34-year-old male with ADPKD was admitted due to abdominal pain for the past 2 days, predominantly in the right flank and associated with temperature reaching up to $99.6^{\circ} \mathrm{F}$. His medical history included the diagnosis of arterial hypertension and chronic kidney disease stage IIIB (CKD-EPI of $37 \mathrm{~mL} / \mathrm{min} / 1.73 \mathrm{~m}^{2}$ ) as well as an episode of intestinal perforation followed by enterectomy in 2013. The patient had been hospitalized twice during the previous 2 months due to UTIs associated with CI and renal function decline. He clinically improved in both occasions following treatment with IV linezolid $600 \mathrm{mg}$ bid plus meropenem $1 \mathrm{~g}$ tid for 6 weeks, being discharged to complete antibiotic treatment on a homecare basis.

His serum creatinine was $2.3 \mathrm{mg} / \mathrm{dL}$ on admission, white blood cell count was $9040 / \mathrm{mm}^{3}$ and CRP 102.9 $\mathrm{mg} / \mathrm{L}$, while urinalysis showed 49,000 leucocytes/mL and 5000 erythrocytes/mL (Table 1). CT scan revealed obstructive acute abdomen secondary to bridles, pneumoperitoneum and gas bubbles in fat tissue adjacent to the transverse colon and hepatic angle. He was therefore submitted to laparotomy, bridle lysis and cavity washing. The surgical intervention was successful however CRP levels remained high, supporting the performance of PET-CT. Enlarged kidneys with multiple cysts containing homogeneous liquid or heterogeneous dense hyperproteic material were detected (Fig. 1g). This imaging assessment also revealed perirenal fascia thickening and high FDG uptake in a left $6.9 \times 5.5 \times 4.1-\mathrm{cm}$ exophytic cyst, establishing the diagnosis of renal CI (Fig. 1h). CTguided percutaneous cyst drainage allowed culture of its content, leading to isolation and diagnosis of Candida albicans CI. Blood cultures were negative. Following treatment with fluconazole for 6 weeks, the patient evolved with full clinical and laboratorial recovery, requiring no additional invasive procedures.

\section{Discussion and conclusion}

Renal and/or hepatic CI is a common complication in ADPKD, with an incidence of approximately 0.01 episode/patient/year $[11,15]$. Several of its aspects, however, remain not well defined, including diagnostic criteria and methods as well as treatment options and 
regimens $[6,9,12]$. Previous studies propose that a definite diagnosis of $\mathrm{CI}$ should be based on cyst aspirate showing neutrophil debris and/or a pathogenic microorganism $[6,16]$. With minor variations, a common algorithm defines as likely $\mathrm{CI}$ the presence of fever (temperature $>38.5^{\circ} \mathrm{C}$ for $>3$ days); abdominal pain (particularly a palpable area of renal or liver tenderness); CRP $>50 \mathrm{mg} / \mathrm{L}$, and absence of recent, significant intracystic bleeding or other causes of fever $[1,3,11,16,17]$. Since positive cyst cultures are usually not available, likely $\mathrm{CI}$ is far more prevalent than definite $\mathrm{CI}$ in the clinical scenario. Some reports detected a high prevalence of cyst infection among patients with advanced stages of chronic kidney disease. Indeed, the first case series using PET-CT to investigate cyst infection in ADPKD reported that 12 out $36(33,3 \%)$ patients were on dialysis support [11], a finding in accordance with our previous report that identified 6 out 24 patients in this condition [10]. Of note, neither of the two currently reported cases developed such a complication while on dialysis.

Antibiotic treatment algorithms targeting $\mathrm{CI}$ are not robustly defined, since the infectious agent is isolated in a minority of cases. Jouret et al. [8] confirmed the pathogenic bacteria with cyst fluid cultures in only 3 of the 27 analyzed likely CI episodes while Sallee et al. [11] reported five positive cyst cultures among 41 evaluated $\mathrm{CI}$ events. In this scenario, different antibiotic approaches have been proposed and used, however none of them includes antifungal drugs as part of initial and second-line regimens. Based on their appropriate concentration within the cyst and antibiotic spectrum against gram-negative bacteria, currently proposed schemes classically include fluoroquinolones, particularly ciprofloxacin or levofloxacin $[1,3,6$, $7,9]$. Failure to improve with antibiotics, however, should raise the suspicion of a fungal infection. There is no consensus, nevertheless, whether routine empirical antifungal therapy should be administered in non-neutropenic patients, even if they are critically ill, since most studies in this specific group have not shown a survival benefit [18]. In our cases, however, both patients had risk factors accepted as criteria for empirical antifungal therapy. Invasive candidiasis is a critical condition associated with mortality in the same level as septic shock (40-60\% mortality-rate). In this context, the presence of well-defined risk factors for this condition should always be evaluated by the designated clinician, especially in an inpatient setting. Identifying risk factors may lead to a quick and accurate diagnosis in clinical scenarios where invasive fungemia is unexpected or isn't contemplated in initial guidelines, such as in the described cases. Upon admission, patient 1 presented important risk factors such as Candida colonization, exposure to antibiotics and use of a double-J catheter. Patient 2, in turn, presented long hospital stay, previous antimicrobial therapy and an emergency gastrointestinal surgical procedure. Other potential risk factors include hemodialysis, necrotizing pancreatitis, central venous catheter, total parenteral nutrition, diabetes mellitus and immunodeficiency (neutropenia $<500$ neutrophils/ $\mathrm{mL}$, hematologic malignancy, chemotherapy, immunosuppressant drugs) $[19,20]$. A fundamental point, moreover, is not to underestimate the diagnostic value of cyst puncture/culture in difficult cases. In intra-abdominal candidiasis, only $4-20 \%$ of cases will be candidaemic [21]. To optimize blood culture sensitivity, 40-60-mL blood collection is recommended in adults.

Candida spp. grow very well in standard blood culture broths [22]. The detection of fungal antigen has been a useful approach to the presumptive diagnosis of invasive fungal infection in populations other than ADPKD. In patients with blood-culturenegative invasive candidiasis, mainly with intraabdominal infection, the $1,3-\beta-\mathrm{D}$-glucan assay presented a sensitivity of $88 \%$ [23]. Some false-positive results, however, have been reported due to crossreactions with certain hemodialysis filters, a caveat that could limit its application in patients undergoing hemodialysis support [24, 25].

Only less than $5 \%$ of Candida albicans isolates are resistant to azole [26]. This finding was corroborated by a retrospective study that analyzed the susceptibility of 153 Candida albicans isolates to three different antifungal drugs [27]. Only $1 \%$ of these isolates were resistant to fluconazole and $3 \%$ presented dose-dependent susceptibility; $96 \%$ of them, therefore, were susceptible to fluconazole. This same study showed that all isolates were susceptible to anidulafungin and only one of the 153 was resistant to amphotericin $\mathrm{B}$. We chose, however, not to attempt these therapies in our first case due to the following reasons: 1) In a previously reported C. kruzei kidney cyst infection, none of the six analyzed cysts presented amphotericin B levels that reached the MIC level for the C. krusei recovered isolate [13]. The patient from this case report was, in fact, eventually submitted to bilateral nephrectomy; and 2) It is important to point out that none of the echinocandins are excreted in the urine as an active drug, therefore these drugs are not contemplated in Candida urinary tract infection treatment algorithms [28].

Notably, we now report two cases of fungal CI, a very unusual form of this complication. To our knowledge, these are the first two documented cases of Candida albicans renal CI; one with successful response to antifungal therapy and another with no improvement following this therapeutic approach, requiring nephrectomy to be resolved. Our report highlights fungi as potential etiologic agents for CI in ADPKD and the importance of including this possibility within the differential diagnosis 
in specific circumstances and clinical courses. Immunodeficient patients, previous prolonged antibiotic use and Candida colonization should call attention to this potential diagnosis. In addition, the identification of two cases of Candida albicans renal CI by our group raises the possibility that fungal $\mathrm{CI}$ may not be as rare as currently assumed. The relevance of such considerations is particularly high given that first and second-line antibiotic regimens currently applied to $\mathrm{CI}$ in ADPKD do not include antifungal agents. The efficacy of antifungal therapeutic strategies according to the identified specimen and its susceptibility and, most importantly, their corresponding intracystic antifungal levels, however, remain to be established.

\section{Abbreviations \\ ADPKD: Autosomal Dominant Polycystic Kidney Disease; AmB: Amphotericin B; Cl: Cyst Infection; CRP: C-reactive protein; CT: Computed Tomography; FDG: F-18 $\left({ }^{18} \mathrm{~F}\right.$ ) Fluor-deoxi-glucose; MRI: Magnetic Resonance Imaging; PET- CT: Positron Emission Tomography/Computed Tomography; UTI: Urinary Tract Infection}

\section{Acknowledgements}

We thank the University of São Paulo Medical Center and Oswaldo Cruz German Hospital for their support to our work.

\section{Authors' contributions}

LO provided clinical care for the patient, performed literature searches, drafted and revised the article; VAHS provided clinical care for the patient and revised the article; PDN provided clinical care for the patient, performed literature searches, drafted and revised the article; BEB provided clinical care for the patient, performed literature searches, drafted and revised the article; APN provided clinical care for the patient and revised the article; FTF provided clinical care for the patient and revised the article; EHW provided clinical care for the patient and revised the article; AW provided clinical care for the patient and revised the article; MCSA provided clinical care for the patient, performed literature searches, drafted and revised the article; LAT provided histologic diagnosis for the patient and revised the article; PRC provided clinical care for the patient, performed literature searches and revised the article; LFO provided clinical care for the patient, performed literature searches, drafted and revised the article. All authors have read and approved the manuscript.

\section{Funding}

There was no specific financial support to this report.

\section{Availability of data and materials}

All meaningful data generated or analyzed in this study are included in the manuscript.

\section{Ethics approval and consent to participate}

Not applicable.

\section{Consent for publication}

Written consent to publish this information was obtained from study participants or their relatives.

\section{Competing interests}

The authors declare that they have no competing interests.

\section{Author details}

'Department of Medicine, Division of Nephrology, University of São Paulo School of Medicine, Avenida Doutor Arnaldo, 455 - Sala 4304, São Paulo, SP 01246-903, Brazil. ${ }^{2}$ Nephrology and Internal Medicine Service, Oswaldo Cruz German Hospital, São Paulo, Brazil. ${ }^{3}$ Division of Infectious Diseases, University of São Paulo School of Medicine, São Paulo, Brazil. ${ }^{4}$ Division of Pathology, University of São Paulo School of Medicine, São Paulo, Brazil.
Received: 1 May 2019 Accepted: 6 September 2019

Published online: 29 October 2019

\section{References}

1. Chebib FT, Torres VE. Autosomal dominant polycystic kidney disease: Core curriculum 2016. Am J Kidney Dis. 2016;67(5):792-810.

2. Willey CJ, Blais JD, Hall AK, et al. Prevalence of autosomal dominant polycystic kidney disease in the European Union. Nephrol Dial Transplant. 2017:32(8):1356-63

3. Chapman AB, Devuyst O, Eckardt KU, et al. Autosomal-dominant polycystic kidney disease (ADPKD): executive summary from a kidney disease: improving global outcomes (KDIGO) controversies conference. Kidney Int. 2015;88(1):17-27.

4. Kwona HW, Lee HY, Hwang YH, et al. Diagnostic performance of 18F-FDGlabeled white blood cell PET/CT for cyst infection in patients with autosomal dominant polycystic kidney disease: a prospective study. Nucl Med Commun. 2016;37(5):493-8.

5. Schwab SJ, Bander SJ, Klahr S, et al. Renal infection in autosomal dominant polycystic kidney disease. Am J Med. 1987:82(4):714-8.

6. Lantinga MA, Drenth JPH, Gevers TJG. Diagnostic criteria in renal and hepatic cyst infection. Nephrol Dial Transplant. 2015;30(5):744-51.

7. Lantinga MA, Casteleijn NF, Geudens A, et al. Management of renal cyst infection in patients with autosomal dominant polycystic kidney disease: a systematic review. Nephrol Dial Transplant. 2017;32(1):144-50.

8. Jouret F, Lhommel R. Devuyst. O, et al. diagnosis of cyst infection in patients with autosomal dominant polycystic kidney disease: attributes and limitations of the current modalities. Nephrol Dial Transplant. 2012;27(10): 3746-51.

9. Suwabe T, Araoka H, Ubara Y, et al. Cyst infection in autosomal dominant polycystic kidney disease: causative microorganisms and susceptibility to lipid-soluble antibiotics. Eur J Clin Microbiol Infect Dis. 2015;34(7):1369-79.

10. Balbo BEP, Sapienza MT, Ono CR, et al. Cyst infection in hospital-admitted autosomal dominant polycystic kidney disease patients is predominantly multifocal and associated with kidney and liver volume. Braz J Med Biol Res. 2014:47(7):584-93

11. Sallée M, Rafat C, Zahar J, et al. Cyst infections in patients with autosomal dominant polycystic kidney disease. Clin J Am Soc Nephrol. 2009;4(7):1183-9.

12. Jouret $F$, Lhommel $R$, Beguin $C$, et al. Positron-emission computed tomography in cyst infection diagnosis in patients with autosomal dominant polycystic kidney disease. Clin J Am Soc Nephrol. 2011;6(7): $1644-50$.

13. Hepburn MJ, Pennick GJ, Sutton DA, et al. Candida krusei renal cyst infection and measurement of amphotericin B levels in cystic fluid in a patient receiving AmBisome. Med Mycol. 2003;41(2):163-5.

14. Padhye AA, Verghese $S$, Ravichandran P, et al. Trichosporon loubieri infection in a patient with adult polycystic kidney disease. J Clin Microbiol. 2003:41(1):479-82.

15. Bobot $\mathrm{M}$, Ghez $\mathrm{C}$, Gondouin $\mathrm{B}$, et al. Diagnostic performance of $\left[{ }^{18} \mathrm{~F}\right]$ fluorodeoxyglucose positron emission tomography - computed tomography in cyst infection in patients with autosomal dominant polycystic kidney disease. Clin Microbiol Infect. 2016;22(1):71-7.

16. Lanktree MB, Chapman AB. New treatment paradigms for ADPKD: moving towards precision medicine. Nat Rev Nephrol. 2017;13(12):750-68.

17. Lantinga MA, Darding AJM, de Sévaux RGL, et al. International multispecialty Delphi survey: identification of diagnostic criteria for hepatic and renal cyst infection. Nephron. 2016;134(4):205-14.

18. Cortegiani A, Russotto V, Maggiore A, et al. Antifungal agents for preventing fungal infections in non-neutropenic critically ill patients. Cochrane Database Syst Rev. 2016;16(1):CD004920.

19. Eggimann P, Garbino J, Pittet D. Epidemiology of Candida species infections in critically ill non-immunosuppressed patients. Lancet Infect Dis. 2003;3(11): 685-702.

20. Colombo AL, de Almeida Júnior JN, Slavin MA, et al. Candida and invasive mould diseases in non-neutropenic critically ill patients and patients with haematological cancer. Lancet Infect Dis. 2017;17(11):e344-56.

21. Chamilos $G$, Luna $M$, Lewis $R E$, et al. Invasive fungal infections in patients with hematologic malignancies in a tertiary care cancer center: an autopsy study over a 15-year period (1989-2003). Haematologica. 2006;91(7):986-9.

22. Miller JM, Binnicker MJ, Campbell S, et al. A quide to utilization of the microbiology Laboratory for Diagnosis of infectious diseases: 2018 update 
by the Infectious Diseases Society of America and the American Society for Microbiology. Clin Infect Dis. 2018;67(6):e1-e94.

23. Nguyen MH, Wissel MC, Shields RK, et al. Performance of Candida real-time polymerase chain reaction, $\beta$-D-glucan assay, and blood cultures in the diagnosis of invasive candidiasis. Clin Infect Dis. 2012;54(9):1240-8.

24. Theel ES, Doern CD. $\beta$-D-glucan testing is important for diagnosis of invasive fungal infections. J Clin Microbiol. 2013;51(11):3478-83.

25. Kanda H, Kubo K, Hamasaki K, et al. Influence of various hemodialysis membranes on the plasma (1-->3)-beta-D-glucan level. Kidney Int. 2001; 60(1):319-23.

26. Pfaller MA, Diekema DJ, Gibbs D, et al. Results from the ARTEMIS DISK global antifungal surveillance study, 1997 to 2005: an 8.5-year analysis of susceptibilities of Candida species and other yeast species to fluconazole and voriconazole determined by CLSI standardized disk diffusion testing. J Clin Microbiol. 2007:45(6):1735-45.

27. Mattei AS, Alves SH, Mario DA, et al. Susceptibility of Candida albicans blood isolates to 3 antifungal drugs: retrospective study in Rio Grande do Sul, Brazil, 1999-2009. Rev Iberoam Micol. 2013;30(4):243-7.

28. Kauffman CA. Diagnosis and management of fungal urinary tract infection. Infect Dis Clin N Am. 2014;28(1):61-74.

\section{Publisher's Note}

Springer Nature remains neutral with regard to jurisdictional claims in published maps and institutional affiliations.

Ready to submit your research? Choose BMC and benefit from:

- fast, convenient online submission

- thorough peer review by experienced researchers in your field

- rapid publication on acceptance

- support for research data, including large and complex data types

- gold Open Access which fosters wider collaboration and increased citations

- maximum visibility for your research: over $100 \mathrm{M}$ website views per year

At BMC, research is always in progress.

Learn more biomedcentral.com/submissions 